\title{
Changing name: changing prospects for psychosis
}

\author{
D. Kingdon ${ }^{1 *}$, L. Taylor ${ }^{1}, K . M^{1}$ and Y. Kinoshita ${ }^{2}$ \\ ${ }^{1}$ University of Southampton, Southampton, UK \\ 2 Shinshu University School of Medicine, Nagano, Japan
}

\begin{abstract}
Names matter! Schizophrenia has negative associations which impede individual recovery and induce societal and selfstigmatization. Alternatives have been proposed and are worthy of debate; changes made in Japan have generally been considered successful. The group of 'schizophrenia and other psychoses' could be further differentiated based on the major social factors identified, i.e. drug misuse and the effects of severe childhood trauma. The use of appropriate International Classification of Diseases (ICD) coding and definitions could usefully differentiate these groups - the former is a drug-induced psychosis and the latter frequently presents as comorbid schizophrenia and borderline personality disorder (often attracting a diagnosis of schizoaffective disorder). The current established differentiation between early onset ('stress-sensitive' - 'Kraepelinian' schizophrenia) and later onset (DSM5 delusional disorder, i.e. with 'nonbizarreness' criterion removed) psychosis may also be worthy of further investigation to establish validity and reliability. Psychosocially descriptive terms have been found to be more acceptable to patients and perceived as less stigmatizing by others. Subgroups of psychosis with greater homogeneity would benefit research, clinical and therapeutic practice and public understanding, attitudes and behaviour.
\end{abstract}

First published online 10 September 2013

Key words: Classification, psychosis, schizophrenia, social factors.

The term, schizophrenia, is now a century old and its readoption by the Diagnostic and Statistical Manual (Fifth Revision - DSM-5) means that it will live on at least until DSM-5.1. However, has the time come for its replacement? The term has become highly stigmatized and associated with concepts of violence, lack of hope and deterioration which work against attempts to further the concept of personal recovery. The calls for its abandonment have therefore been growing over the past decade (Kingdon, 2007; Hammersley \& McLaughlin, 2013).

The argument has been made that names do not matter (Lieberman \& First, 2007) as changing them does not address 'the core problem of stigmatization - public ignorance and fear' and that stigma will move with the condition irrespective of what it is called. However, this seems contrary to experience in other areas: terms used over the past century such as idiot, spastic and manic depressive have all been changed with success. This has not eradicated the stigma attached to the conditions but has provided more generally acceptable terms. Certainly any return to the use of the previous terminology is not contemplated and use of it is now seen as insulting and derogatory.

\footnotetext{
${ }^{*}$ Address for correspondence: Professor D. Kingdon, University of Southampton, College Keep, 4-12 Terminus Terrace, Southampton SO14 3DT, UK.

(Email: dgk@soton.ac.uk)
}

There is also some suggestion that patients who accept that they have the illness, schizophrenia, are also more likely to be depressed (Rathod et al. 2005) and biomedical psychoeducation focusing on accepting illness has been found to be associated with suicidal thinking (Cunningham Owens et al. 2001).

The protestation against changes also ignores completely the importance that is attached to names as exemplified by the existence of a very active, wellfunded and extensive marketing industry spending considerable resources in time and money, naming everything from washing powders to confectionary and also new antipsychotics (Kingdon et al. 2007). Why were names like Abilify and Zyrexa chosen after spending significant amounts of time and money? One reason might be that the pharmaceutical industry knew that people, including psychiatrists, are more likely, given a choice, to choose from the beginning and end of lists. Those people who labour under the effects of the misconceptions surrounding terms such as schizophrenia deserve more consideration to be given to this issue.

Schizophrenia is a hybrid of Greek and Latin incorporating words conveying the sense of 'splitting' and 'mind'. Is this really how we would want to convey what psychosis is? It fails to capture the nature of the disorder although the diverse presentations do make this difficult. In the International Classification of Diseases (ICD) and Diagnostic and Statistical 
Manuals, schizophrenia has been linked with 'other psychoses' and this latter broad umbrella term has some advantages in being in common use and recognizable. It broadly conveys the underlying concept that there is a problem with attaching meaning or significance to events or perceptions. Alternative terms proposed have been Bleulers disease (Blaj, 2007), Salience dysregulation syndrome (George, 2009; van Os, 2009a, b) and Integration Disorder. There are no published evaluations of the initial two alternatives as yet but there has been work on the latter.

After a campaign by patients and their carers in Japan (Kim \& Neurology, 2002; Desapriya \& Nobutada, 2003), the Japanese Society of Psychiatry and Neurology changed the old term for the disorder, 'Seishin Bunretsu Byo' ('mind-split-disease'), into the new term of 'Togo Shitcho Sho' ('integration disorder'). The main reasons given for the renaming were: 'the ambiguity of the old term, the recent advances in schizophrenia research, and the deep-rooted negative image of schizophrenia, in part related to the long-term inhumane treatment of most people with the disorder in the past. The renaming was associated with the shift from the Kraepelinian disease concept to the vulnerability-stress model'.

A pan-Japanese survey carried out 7 months after renaming found that the old term had been replaced by the new one in $78 \%$ of cases. Renaming coincided with an increase in the percentage of cases in which patients were informed of the diagnosis from 36.7\% to $69.7 \%$ over the next 3 years. Eighty-six per cent of psychiatrists in a survey in the Miyagi prefecture expressed the view that the new term was more suitable and it has informed treatment guidelines (Sato, 2006). Further work using the Implicit Association Test, designed to minimize bias, assessed the impact of the renaming on the stereotype of schizophrenia in young people. The old term was strongly associated with 'criminal', and this association became significantly weaker with the new term. (Takahashi et al. 2009).

The Director of the National Institute of Mental Health (NIMH) has taken a more radical stance in criticizing DSM-5 and has announced that a new approach is needed to classification (Cuthbert \& Insel, 2013). He is emphasizing the development of genetic and biological markers although, as the DSM-5 Task Force lead commented, there are none currently known despite extensive search for them. NIMH are developing Research Domain Criteria (RDoC) linking brain circuits with dysfunction but can currently find no place within them for developmental and environmental markers. However, research on social and psychological markers for psychosis has been transforming conceptualization and research. There is now very clear evidence for much higher incidences of childhood trauma (Bentall et al. 2012) and drug misuse (Manrique-Garcia et al. 2012) in psychosis.

The British Psychological Association has recently made a call to abandon classification altogether and focus on individual formulation as patient descriptors. However, while formulation is essential for communication and treatment, it does not seem to meet needs for names ('labels') in day-to-day usage. It is empowering for patients and carers to be able to seek information on treatment and prognosis but is hard to conceptualize how this would occur if they only had their individual formulation. Payment for services and welfare benefits also require short labels, which can be understood by the relevant agencies. Grouping of formulations together is also necessary to research conditions in a way that can be generalized. Focusing on individual symptoms has also been proposed and some, e.g. hearing voices, seem to lend themselves to this approach and others, e.g. delusions of control, seem less so. Voices, as an example, can also differ in a range of different ways that seem more directly related to conditions such as depression or mania.

A relevant issue is whether we are describing as Bleuler originally said, a 'group of Schizophrenias' or one entity. DSM-5 has made changes to the 'Schizophrenia and other disorders' chapter by removing the subgroups of schizophrenia, which had previously existed in classifications. This is justified as they were not used much in research or clinical work and have lacked validity and reliability (Braff et al. 2013).

As described above however, there are relevant social and psychological factors that could form the basis for classification. Both childhood trauma and drug misuse have been found to be clinically relevant to psychological treatment and ways of working with them have been described in cognitive behaviour therapy manuals (Kingdon \& Turkington, 2005). Descriptions of 'traumatic psychosis' (Mansell \& Morrison, 2007) and 'drug-induced/precipitated psychosis' now exist and a structured clinical interview (Kinoshita et al. 2012) has been developed to differentiate them from each other and from 'early' and 'late-onset' psychosis.

Although this work has developed out of clinical experience, there is also a strong body of research evidence that was neglected during the recent debates around classification of psychosis. There is a group of patients who meet DSM IV criteria for both borderline personality disorder and schizophrenia and have very high levels of childhood abuse, i.e. a 'traumatic psychosis' (Kingdon et al. 2010). Patients were 
recruited from mental health services with diagnoses of schizophrenia or borderline personality disorder $(n=111)$ and interviewed using the sections of the Structured Clinical Interviews 1 and 2 relevant to diagnosis of these conditions. A total of $53 \%$ met criteria for schizophrenia alone; $30 \%$ for borderline personality disorder alone and $17 \%$ of the sample met criteria for both diagnoses, i.e. $24 \%$ of the total who met criteria for schizophrenia also met criteria for borderline personality disorder. In this comorbid group, 82\% reported severe emotional abuse in childhood and $44 \%$ severe sexual abuse compared with 92 and $67 \%$ in the borderline only group. Many of these comorbid patients had previously attracted a diagnosis of schizoaffective disorder. Schizoaffective disorder survived in DSM-5 - just, and after much consideration. It has been used in patients with psychosis who seem to be hovering near the boundary with bipolar disorder or are very depressed and its reliability has been poor. An attempt has been made to improve this by emphasizing the connection of mood with psychotic features as primary and that the diagnosis should apply only where affective components have been present for substantial periods of the illness time course. The group of patients who remain, will include those in whom mood fluctuations, dysregulation, seem particularly prominent and who may therefore be the group described above who have comorbid borderline personality disorder (Kingdon et al. 2010).

The drug-related groups have emerged since the Second World War when transient drug-induced and then more persistent psychotic episodes caused by amphetamines were reported in Japan (Tomiyama, 1990). Since that time, it has become increasingly apparent that amphetamines, cocaine, Lysergic acid diethylamide (LSD) and stronger forms of cannabis can also lead to persistent illnesses. There is evidence that this group differ from other patients with the diagnosis of schizophrenia in that they have more positive and less negative symptoms, are more sociable and at higher risk of aggressive behaviour towards others (Miles et al. 2003). They may be less likely to respond to antipsychotics (Green et al. 2007) and cognitive behaviour therapy (Barrowclough et al. 2010) although low-to-moderate levels of usage may not interfere with the latter (Naeem et al. 2005). Substance induced psychotic disorder is a category in DSM- 5 but unfortunately can only be used if the episode lasts less that 1 month after discontinuation of substances. ICD10 does not specify a time limit so allows for the categorization as F10-19 (which determines the substance involved) with qualifying:

'.5 Psychotic disorder. A cluster of psychotic phenomena that occur during or following psychoactive substance use but that are not explained on the basis of acute intoxication alone and do not form part of a withdrawal state. The disorder is characterized by hallucinations (typically auditory, but often in more than one sensory modality), perceptual distortions, delusions (often of a paranoid or persecutory nature), psychomotor disturbances (excitement or stupor), and an abnormal affect, which may range from intense fear to ecstasy. The sensorium is usually clear but some degree of clouding of consciousness, though not severe confusion, may be present'

This does however seem to be an infrequently used category.

There is also a case to be made for separating early and late onset psychoses and this has been assisted by removal in DSM-5 of the 'bizarreness' criterion. There has been a change in the criteria for delusions which no longer need to be bizarre in schizophrenia or nonbizarre in delusional disorder. This certainly reflects experience of working using cognitive therapy with patients with psychosis that bizarreness often reflects the observer's lack of understanding about the sources from and ways in which beliefs developed. Bizarre expressions may be attached to feelings which the patient does not understand, for example, being electrocuted as an explanation for the experience of a startle reflex or from paraesthesiae 'tingling' associated with hyperventilation from anxiety. It can also come from a lack of social knowledge, e.g. a patient feared the moon was going to fall in on him due to his lack of knowledge of simple physics. Collaborative working can lead to mutual understanding of these processes even if it does not result in agreement on their significance. It makes determining what is a bizarre or a non-bizarre delusion especially difficult to determine. The late onset group had received diagnoses of delusional disorder, or where bizarre delusions were present, schizophrenia. They have often developed anxiety and sometimes depression from stressful events which may lead through a 'delusional mood' to interpretation in a frankly delusional manner and subsequent systematization. This 'search for meaning' and arrival at a delusional conclusion can alleviate this anxiety but be strongly reinforcing to the belief. This high conviction can seriously impair engagement in treatment. The later onset means that this group have often formed relationships but frequently events such as divorce, separation or distancing within the relationship interfere with the process of reality-testing with confidants. It also means however that they may be more able to survive in society if they default from treatment, as they frequently do. They may still continue to be very ill and lead to disturbance to neighbours, families, housing departments, politicians, police, courts, etc., but not sufficient for them to be returned compulsorily to treatment services. 
Table 1. Psychosocial subgroups

\section{Sensitivity psychosis (early onset)}

Drug-related psychosis

Anxiety psychosis (late onset)

Traumatic psychosis
'My problems began over a period of a few months or even a year or two. I became quite sensitive to stress, which gradually led to interference with what I was doing. This led to increasing confusion and worry and eventually I received treatment. It was or has been difficult to get going again properly - however, hard I try.'

'My problems started after I had taken speed (amphetamines), LSD, cocaine or a lot of cannabis. After that I started to get some problems and received treatment. The problems continued, or came back after settling after the first time this happened. Eventually these problems were happening even when I did not take drugs.'

'When I first received treatment for my problems, I had been having some hassle, stress, and so on, but had become convinced that there was a particular reason behind it all. Unfortunately other people did not agree with me.'

'My problems go back quite a way - maybe even as far as my childhood or soon after - and seem to have something to do with some very unpleasant experiences that I had. Now I seem to get unpleasant voices and maybe also visions - sometimes to do with those experiences.'
The early onset group equate to 'traditional' schizophrenia developing in adolescence and early adulthood - the term 'sensitivity' has been used to denote the sensitivity to stress and the interpersonal characteristics that this group tend to show (Myin-Germeys et al. 2005). It is a term that has proved very popular and explanatory with patients and their carers. There has however been little research into distinctions, or otherwise, between these early and late groups and such a study is currently underway.

Dudley et al. (2009) have taken an interesting approach to this problem by investigating what factors people themselves believed led to the onset of their psychosis. A $Q$ set of potential causes for psychosis was identified from a literature search and interviews with people with differing experiences of psychosis. From this, 58 potential causes of psychosis were identified. Twenty-one people who had experienced a psychotic breakdown then ranked these explanations as possible causes. Using Principal Components Analysis four main factors were identified as perceived causal factors for the onset of psychosis. These factors were described as: (a) drug usage, (b) traumatic experiences in adulthood, (c) personal sensitivity and (d) developmental vulnerabilities - very similar to the groupings proposed previously.

Psychosis is a term that is not without stigma but was preferred, when qualified with descriptive terms, in a study involving patients and mental health staff (Kingdon et al. 2008). Patients who met criteria for schizophrenia, schizoaffective or delusional disorder were asked to select the term which they felt best described their condition. The six terms were offered with a brief description. They were schizophrenia, 'none of these' and new terms based on the distinctions described above: sensitivity, drug-related, traumatic and anxiety psychoses (reincarnating Wernicke's term 'anxiety psychosis') (Table 1).

Schizophrenia was chosen by four out of 29 participants, two chose 'none' with the remainder choosing one of the new terms. The community mental health staff and consultant psychiatrists for the individual patients were also asked to do the same exercise and, perhaps surprisingly, also were much more inclined to use the newer terms.

In a separate study, medical students were asked to consider these terms and rate them on a questionnaire that had previously been used to assess stigmatization (Kingdon et al. 2008). There were some striking differences in perceptions of the terms. Overall attitudes were significantly less negative with the alternatives. The students were less negative about the potential for recovery in relation to all the subgroups than for schizophrenia. Concerns about dangerousness were also less prominent with the exception of the drug related group.

A change in terminology could be expected to give a boost to destigmatization programmes and symbolize a change in the way of thinking about the condition. Any such change will need to include assessments with the key audiences - patients, carers and the general public. It would define groupings using existing social factors and, if it led to more homogeneous groups, might assist in the identification of relevant psychological and even biological correlates and interventions.

\section{Financial Support}

This research received no specific grant from any funding agency, commercial or not-for-profit sectors. 


\section{Conflict of Interest}

None.

\section{References}

Barrowclough C, Haddock G, Wykes T, Beardmore R, Conrod P, Craig T, Davies L, Dunn G, Eisner E, Lewis S, Moring J, Steel C, Tarrier N (2010). Integrated motivational interviewing and cognitive behavioural therapy for people with psychosis and comorbid substance misuse: randomised controlled trial. British Medical Journal 341, c6325.

Bentall RP, Wickham S, Shevlin M, Varese F (2012). Do specific early-life adversities lead to specific symptoms of psychosis? A study from the 2007 the adult psychiatric morbidity survey. Schizophrenia Bulletin 38, 734-740.

Blaj A (2007). Bleuler's disease? Re: renaming schizophrenia: the need for evidence. British Medical Journal, Rapid Response.

Braff DL, Ryan J, Rissling AJ, Carpenter WT (2013). Lack of use in the literature from the last 20 years supports dropping traditional schizophrenia subtypes from DSM-5 and ICD-11. Schizophrenia Bulletin 39, 751-753.

Cunningham Owens DG, Carroll A, Fattah S, Clyde Z, Coffey I, Johnstone EC (2001). A randomized, controlled trial of a brief interventional package for schizophrenic out-patients. Acta Psychiatrica Scandinavica 103, 362-369.

Cuthbert BN, Insel TR (2013). Toward the future of psychiatric diagnosis: the seven pillars of RDoC. BMC Medicine 11, 126.

Desapriya E, Nobutada I (2003). Debate over Japanese renaming of term for schizophrenia. Lancet 361, 181.

Dudley R, Siitarinen J, James I, Dodgson G (2009). What do people with psychosis think caused their psychosis? A Q methodology study. Behavioural and Cognitive Psychotherapy $37,11-24$.

George B (2009). Salience dysregulation syndrome: a patient's view. British Journal of Psychiatry 194, 467.

Green AI, Drake RE, Brunette MF, Noordsy DL (2007). Schizophrenia and co-occurring substance use disorder. American Journal of Psychiatry 164, 402-408.

Hammersley P, McLaughlin T (2013). Campaign for the Abolition of Schizophrenia Label. Retrieved 9 June 2013 http://www.asylumonline.net/resources/ campaign-for-the-abolition-of-schizophrenia-label/.

Kim Y (2002). Renaming the term schizophrenia in Japan. Lancet 360, 879.

Kingdon D (2007). Down with schizophrenia. New Scientist 2625, 35-53.

Kingdon D, Turkington D (2005). Cognitive Therapy for Schizophrenia. Guilford: New York.

Kingdon D, Vincent S, Kinoshita Y, Turkington D (2008). Destigmatising schizophrenia: does changing terminology reduce negative attitudes? Psychiatric Bulletin 32, 419-422.

Kingdon DG, Kinoshita Y, Naeem F, Swelam M, Hansen L, Vincent S, Rathod S (2007). Schizophrenia can and should be renamed. British Medical Journal 334, 221-222.
Kingdon DG, Ashcroft K, Bhandari B, Gleeson S, Warikoo N, Symons M, Taylor L, Lucas E, Mahendra R, Ghosh S, Mason A, Badrakalimuthu R, Hepworth C, Read J, Mehta R (2010). Schizophrenia and borderline personality disorder: similarities and differences in the experience of auditory hallucinations, paranoia, and childhood trauma. Journal of Nervous and Mental Diseases 198, 399-403.

Kinoshita Y, Kingdon D, Kinoshita K, Sarafudheen S, Umadi D, Dayson D, Hansen L, Rathod S, Ibbotson RB, Turkington D, Furukawa TA (2012). A semi-structured clinical interview for psychosis sub-groups (SCIPS): development and psychometric properties. Social Psychiatry and Psychiatric Epidemiology 47, 563-580.

Lieberman JA, First MB (2007). Renaming schizophrenia. British Medical Journal 334, 108.

Manrique-Garcia E, Zammit S, Dalman C, Hemmingsson T, Andreasson S, Allebeck P (2012). Cannabis, schizophrenia and other non-affective psychoses: 35 years of follow-up of a population-based cohort. Psychological Medicine 42, 1321-1328.

Mansell W, Morrison A (2007). Trauma and Psychosis. Psychology Press: London.

Miles H, Johnson S, Amponsah-Afuwape S, Finch E, Leese M, Thornicroft G (2003). Characteristics of subgroups of individuals with psychotic illness and a comorbid substance use disorder. Psychiatric Services 54, 554-561.

Myin-Germeys I, Delespaul P, Van Os J (2005). Behavioural sensitization to daily life stress in psychosis. Psychological Medicine 35, 733-741.

Naeem F, Kingdon D, Turkington D (2005). Cognitive behavior therapy for schizophrenia in patients with mild to moderate substance misuse problems. Cognitive Behavioral Therapy 34, 207-215.

Rathod S, Kingdon DG, Smith P, Turkington D (2005). Insight into schizophrenia: the effects of cognitive behavioural therapy on the components of insight and association with sociodemographics - data on a previously published randomised controlled trial. Schizophrenia Research 5, 211-219.

Sato M (2006). Renaming schizophrenia: a Japanese perspective. World Psychiatry 5, 53-55.

Takahashi H, Ideno T, Okubo S, Matsui H, Takemura K, Matsuura M, Kato M, Okubo Y (2009). Impact of changing the Japanese term for 'schizophrenia' for reasons of stereotypical beliefs of schizophrenia in Japanese youth. Schizophrenia Research 112, 149-152.

Tomiyama G (1990). Chronic schizophrenia-like states in methamphetamine psychosis. Japanese Journal of Psychiatry and Neurology 44, 531-9.

Van Os J (2009a). A salience dysregulation syndrome. British Journal of Psychiatry 194, 101-103.

Van Os J (2009b). 'Salience syndrome' replaces 'schizophrenia' in DSM-V and ICD-11: psychiatry's evidence-based entry into the 21st century? Acta Psychiatrica Scandinavica 120, 363-372. 